\title{
Modeling and Design Adaptive Double Neural Network Controller for Eight-Rotor Micro Aircraft Vehicle
}

\author{
Di $\mathrm{Li}^{1}$, Zhihong $\mathrm{Lu}^{1}$ and Xiang-jian Chen ${ }^{2}$ \\ ${ }^{1}$ China Shipbuilding Industry Corporation 723, China \\ ${ }^{2}$ Jiangsu University of Science and Technology, School of computer science and \\ Engineering, China \\ ${ }^{1}$ lidi19821111@163.com, ${ }^{2}$ cxj831209@163.com
}

\begin{abstract}
In this article, a dynamic model of a six degrees of freedom (6 DOF) Eight-Rotor MAV (micro aerial vehicles) is derived on the basis of the Newton-Euler formalism. The derivation comprises determining equations of the motion of the Eight-Rotor MAV in three dimensions and approximating the actuation forces through the modelling of aerodynamic coefficients and electric motor dynamics. For Eight-Rotor MAV is inherently unstable as they are highly sensitive to external perturb, here provides an adaptive double neural network controller for the motion control of the Eight-Rotor MAV autonomous flight. This controller is developed in three parts around each of the variables. The experimental results show that the proposed adaptive double neural network controller outperforms the conventional PID controller due to its fast adaptive qualities in the presence of sensor measurement noise and the parameters variations of Eight-Rotor MAV.
\end{abstract}

Keywords: Six degree of freedom (6 DOF) Eight-Rotor Micro Aircraft Vehicle (MAV), adaptive double neural network controller, autoregressive recurrent neural network structures, self tuning regulator; online training

\section{Introduction}

The recent growth in interest of Micro Aerial Vehicle (MAV) design is a testament to their increasing strategic importance. Small, light, and versatile crafts will dominate the field of reconnaissance, be it military intelligence or search and rescue. MAVs are easier to transport, harder to detect and cheaper to operate. However, it also makes them more unstable, harder to control, and requires greater miniaturization of payload sensors and controller hardware. Traditional MAVs have been miniaturized airplanes due to their greater inherent stability. However, an advance in controller technology has allowed the development of rotorcraft MAVs. The advantages of MAV rotorcraft are often lost when the disadvantages of certain designs are examined. Helicopters, for example, are the most common style of rotorcraft but suffer on the micro-scale duel to their mechanical complexity. Helicopters generate lift thought their single overhead propeller. An additional propeller is located in the tail to counteract the torque form the main propeller. Instead of simple mechanical linkages linked to control surfaces as on an airplane.

Quadrotor [1-5] craft share the same advantages in manoeuvrability with other rotorcraft, but without the mechanical complexity of a helicopter. Instead of a single main rotor and a secondary tail rotor, a Quadrotor has four equal seized rotors. These rotors are not variable-pitch rotors as on a helicopter, instead, the craft is manoeuvred 
by adjusting the relative speed between the individual rotors. This makes the Quadrotor mechanically much simpler, which aids in scaling down to a micro-scale, but this vehicle is a under actuated system which has six inputs in relation to four outputs, which makes the control harder.

Aiming at solving design drawbacks of the Quadrotor MAV, here design a EightRotor MAV which has eight rotors, The eight rotors are arranged as four counterrotating offset pairs mounted at the ends of the four arms, with matched sets of counterrotating rotor blades. Differential thrust from these four equally spaced points make the Eight-Rotor able to manoeuvre quickly and precisely. The off set layout doubles the thrust without increasing the size of the footprint, and naturally eliminates loss of efficiency due to torque compensation. The rotors are optimized for maximum efficiency, low noise, low weight, and high strength. The rotors are mounted on brushless motors designed to direct drive them with maximum power and efficiency. The motors are mounted to the four-arm carbon fibbers frame designed for maximum strength and minimum weight. Eight efficient, electric motors allow the Eight-Rotor to carry heavier and more advanced payloads while providing more safety features than ever before.

The aim of the flight control system is to attain the commands generated by the guidance system and to maintain steady conditions during flight. But the Eight-Rotor MAV is highly susceptible to atmospherics disturbances that deviate they form the designated flight path. In addition, the dynamics displayed if highly nonlinear and time varying. Hence, adaptive flight controllers $[5,6]$ with the ability to achieve desired performance in the presence of external perturbations are essential for automating the Eight-Rotor MAV. In order to achieve this autonomous flight, adaptive flight controllers with the ability to adapt to nonlinear dynamics of the Eight-Rotor MAV are necessary. Thus the main objective of the project is to model this multi-input multioutput nonlinear Eight-Rotor MAV and design adaptive controllers in order to demonstrate their real-time application for Eight-Rotor MAV to achieve autonomous flight.

This paper describes the design and control of the Eight-Rotor MAV based on my previous research [8]. Section2 provides the dynamic modelling for the proposed EightRotor MAV. Section 3 describes the double neural network [9, 10] adaptive control technique, an adaptive neural network controllers based on self-tuning regulatory (STR) are reviewed. The double neural network algorithm is proposed to provide enhanced performance with faster tracking. The stability of the overall adaptive system is analyzed. The overall Eight-Rotor MAV control architecture with the application of a conventional gain based controller and the double neural network controller are presented in Section4. Simulations are carried out; the adaptive double neural network controllers provided the fast adaptation and improved accuracy compared with the conventional PID controller.

\section{Dynamic Modeling of Eight-Rotor MAV}

\subsection{Flight Theory of Eight-Rotor MAV}

The Eight-Rotor is very well modelled with eight rotors in a cross configuration. This cross structure is quite thin and light, however it shows robustness by linking mechanically the motors. Each propeller is connected to the motor through the reduction gears. All the propellers axes of rotation are fixed and parallel. These considerations point out that the structure is quite rigid and the only things that can vary are the propeller speeds. Neither the 
motors nor the reduction gears are fundamental because the movements are directly related just to the propellers velocities can be seen in Table 1 and Figure 1.

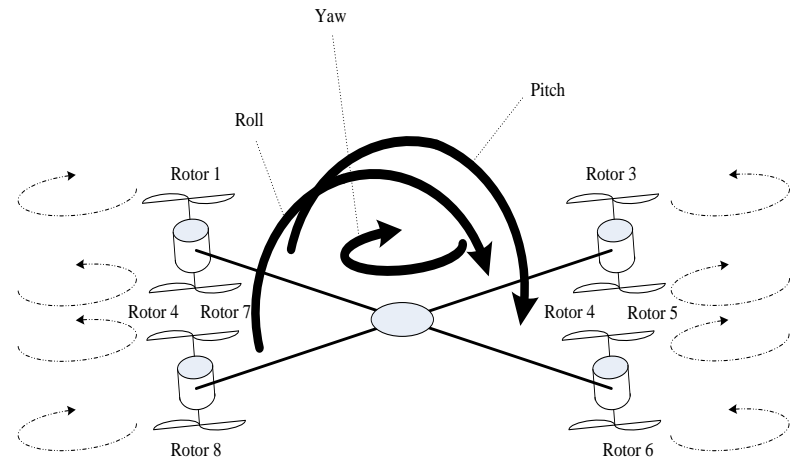

Figure 1. Flight Theory of Eight-Rotor MAV

\section{Table 1. Flight Theory for Eight-Rotor MAV}

\begin{tabular}{|c|c|}
\hline$\Omega_{1}+\Omega_{2}=\Omega_{3}+\Omega_{4}=\Omega_{5}+\Omega_{6}=\Omega$ & $\begin{array}{l}\text { Ešen, Upward Thrust } \Rightarrow \text { No pitch or roll } \\
\text { Torques generated by rotors cancel } \\
\Rightarrow \text { No yaw }\end{array}$ \\
\hline $\begin{array}{l}\left(\Omega_{5}+\Omega_{6}+\Omega_{1}+\Omega_{2}\right)=\left(\Omega_{3}+\Omega_{4}+\Omega\right. \\
\left(\Omega_{1}+\Omega_{2}\right)>\left(\Omega_{5}+\Omega_{6}\right) \\
\left(\Omega_{3}+\Omega_{4}\right)=\left(\Omega_{7}+\Omega_{8}\right)\end{array}$ & $\begin{array}{l}\text { Torques generated by rotors } \\
\text { cancel } \Rightarrow \text { No yaw } \\
\text { More thrust at Rotors } 1,2 \text { than Rotors } 5 \\
, 6 \Rightarrow \text { Pitch } \\
\text { Thrust at Rotors } 3,4=\text { Thrust at Rotors } \\
7,8 \Rightarrow \text { No roll }\end{array}$ \\
\hline $\begin{array}{l}\left(\Omega_{5}+\Omega_{6}+\Omega_{1}+\Omega_{2}\right)=\left(\Omega_{3}+\Omega_{4}+\Omega\right. \\
\left(\Omega_{7}+\Omega_{8}\right)>\left(\Omega_{3}+\Omega_{4}\right) \\
\left(\Omega_{1}+\Omega_{2}\right)=\left(\Omega_{5}+\Omega_{6}\right)\end{array}$ & $\begin{array}{l}\text { Torques generated by rotors } \\
\text { cancel } \Rightarrow \text { No yaw } \\
\text { More thrust at Rotors } 7,8 \text { than Rotors } 3 \\
, 4 \Rightarrow \text { Roll } \\
\text { Thrust at Rotors } 1,2=\text { Thrust at Rotors } \\
5,6 \Rightarrow \text { No pitch }\end{array}$ \\
\hline $\begin{array}{l}\left(\Omega_{3}+\Omega_{4}+\Omega_{7}+\Omega_{8}\right)>\left(\Omega_{5}+\Omega_{6}+\Omega\right. \\
\left(\Omega_{7}+\Omega_{8}\right)=\left(\Omega_{3}+\Omega_{4}\right) \\
\left(\Omega_{1}+\Omega_{2}\right)=\left(\Omega_{5}+\Omega_{6}\right)\end{array}$ & $\begin{array}{l}\text { More torque generated by Rotors } 3,4,7,8 \\
\text { than Rotors } 5,6,1,2 \Rightarrow \text { Yaw } \\
\text { Thrust at Rotors } 7,8=\text { Thrust at Rotors } 3 \\
, 4 \Rightarrow \text { No pitch } \\
\text { Thrust at Rotors } 1,2=\text { Thrust at Rotors } \\
5,6 \Rightarrow \text { No roll }\end{array}$ \\
\hline
\end{tabular}

This can be expanded to move the Eight-Rotor platform at any combination of yaw, roll, and pitch. Because the Eight-Rotor can be controlled by simply varying the speed of 8 motors, it is a very mechanically simple platform and this simplicity would make a very robust system.

\subsection{Eight-Rotor MAV Dynamic Modeling}

The mathematical Eight-Rotor model is based upon the previously developed model [8]. The main aerodynamic effects $U_{i}$ applied by the rotors in the body frame are proportional to the square of the rotor speeds $\Omega_{i}$ : 


$$
\begin{aligned}
& \left(U_{1}=b\left(\left(\Omega_{1}+\Omega_{2}\right)^{2}+\left(\Omega_{4}+\Omega_{3}\right)^{2}+\left(\Omega_{5}+\Omega_{6}\right)^{2}+\left(\Omega_{7}+\Omega_{8}\right)^{2}\right)\right. \\
& U_{2}=b l\left(\left(\Omega_{1}+\Omega_{2}\right)^{2}-\left(\Omega_{5}+\Omega_{6}\right)^{2}\right) \\
& \left\{U_{3}=b l\left(\left(\Omega_{7}+\Omega_{8}\right)^{2}-\left(\Omega_{3}+\Omega_{4}\right)^{2}\right)\right. \\
& U_{4}=d\left(\left(\left(\Omega_{7}+\Omega_{8}\right)^{2}+\left(\Omega_{3}+\Omega_{4}\right)^{2}\right)-\left(\left(\Omega_{1}+\Omega_{2}\right)^{2}+\left(\Omega_{5}+\Omega_{6}\right)^{2}\right)\right) \\
& \Omega=\left(\left(\Omega_{7}+\Omega_{8}\right)^{2}+\left(\Omega_{3}+\Omega_{4}\right)^{2}\right)-\left(\left(\Omega_{1}+\Omega_{2}\right)^{2}+\left(\Omega_{5}+\Omega_{6}\right)^{2}\right)
\end{aligned}
$$

Where $U_{1}$ is the total thrust due to all four rotors, $U_{2}$ is the difference between the thrusts of rotors pair 1-2 and 5-6, $U_{3}$ is the difference between the thrusts of rotors pair 3- and 7-8, and $U_{4}$ is the difference between the thrusts of oppositely spinning motors. The thrust and drag coefficients, $b$ and $d$ respectively, are craft dependent values. The equations of motion in terms of the above applied forces and torques shown in Eq. (2):

$$
\left\{\begin{array}{l}
\dot{u}=(v r-w q)+g \sin \theta \\
\dot{v}=(w p-u r)-g \cos _{\theta} \sin _{\phi} \\
\dot{w}=(u q-v p)-g \cos _{\theta} \sin _{\phi}+\frac{U_{1}}{m} \\
\dot{p}=\frac{I_{y}-I_{z}}{I_{x}} q r-\frac{J_{t p}}{I_{x}} q \Omega+\frac{U_{2}}{I_{x}} \\
\dot{q}=\frac{I_{z}-I_{x}}{I_{y}} p r+\frac{J_{t p}}{I_{y}} p \Omega+\frac{U_{3}}{I_{y}} \\
\dot{r}=\frac{I_{x}-I_{y}}{I z} q r+\frac{U_{4}}{I z}
\end{array}\right.
$$

\section{Adaptive Double Neural Network Controller}

\subsection{Adaptive Self Tuning Regulator Controller}

Direct adaptive control technique has the parameters of the controller updated directly based on the plant response. Gain scheduling for PID controllers [12] is a common adaptive control technique used for various applications. An advantage of the gain scheduling technique is that it allows the parameters to be changed quickly with change in the plant dynamics. However, the application of the technique is limited due to the absence of a learning process.

Indirect adaptive control technique have recursively updated model of the plant which is used to modify the parameters of the controller. In case of neural network based indirect adaptive control, predictions obtained from recursively updated neural network plant model is used to adapt the control laws. A notable architecture available for indirect adaptive control is self turning regulator. One neural network based self tuning regulator is shown in Figure 2. 


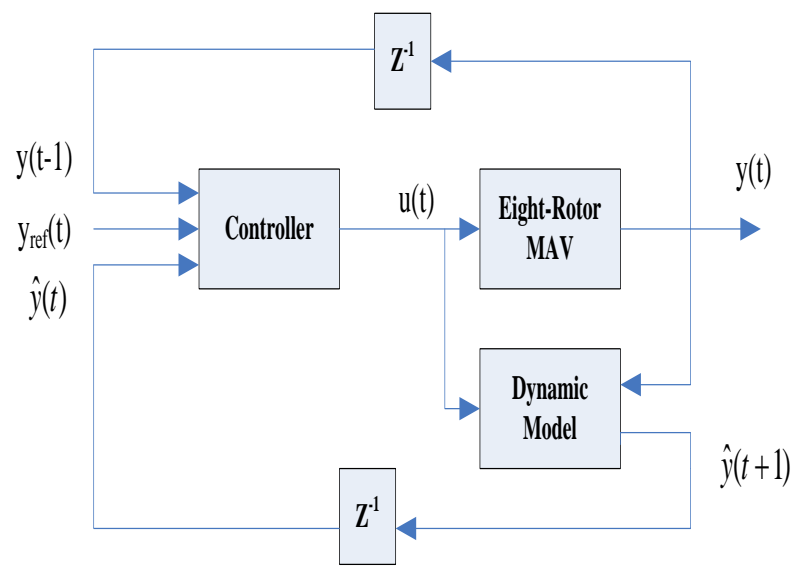

Figure 2. Block Diagram of Self Tuning Regulator Control

The real-time implementation of this controller architecture may not be practical due to the following reasons: The feedback form the identifier model for the iterative change in the controller outputs requires at least one sample time, the computation time to obtain the new set of plant inputs is considerably high for multiple iterations of training; The twofold responsibility of the identifier model may cause ambiguity when both the controller and the identifier require training.

To address this issue related to the classical self-tuning regulatory control a novel double neural network adaptive control architecture is proposed, the neural network self tuning regulatory control technique is modified to include another pre-trained network with the neural network controller for faster training and validation of the controller.

\subsubsection{Adaptive Double Neural Network Controller}

In the work presented here, the neural network self tuning regulators are modified into a double neural network architectures to allow faster adaptations for better performance. In the proposed control architecture, a pre-trained offline model network is concatenated with a controller network internally. The internal model network is trained with actuator inputs and steady state outputs of the nonlinear plant. A schematic block diagram of the proposed double neural network architecture is shown in Figure 3. Where the controller outputs are validated against an external model at every instant of time, the DNN architecture has the internal model network which provides corrections to the controller outputs at every training iteration. Controller network outputs upon completion of training are validated against an external plant identifier model that is trained online. The online trained model predicts the plant behaviours corresponding to the inputs from the double neural network controller [11]. This predicted output is compared with the commanded reference input and suitable modifications to the weights are performed to obtain the desired plant outputs at every instant of time. This effectively provides two feedback loops for the controller network, one by the internal model network at every iteration and another by the external online trained model at every sample time. 


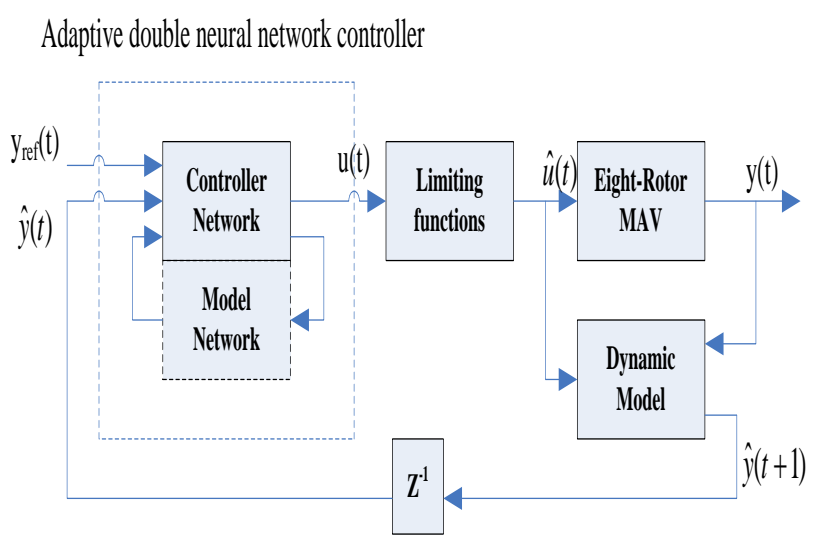

\section{Figure 3. Block Diagram of Adaptive Control System with Adaptive Double Neural Network Controller}

\subsubsection{Double Neural Network Controller Structure}

The model network and the controller network have autoregressive recurrent neural network structures. The entire online controller training process occurs within a single sample period. The reference input $y_{\text {ref }}(t)$, online plant model output $y_{m}(t)$ and the autoregressive terms form the external inputs to the controller network. The model network output $y_{\text {in }}(t)$ forms the internal input to the controller network. Saturation $\mathrm{m}$ limits on the absolute values of the controller outputs and limiting functions on the rate of change of these outputs are imposed to provide suitable bounds on the controller outputs. The outputs from the controller $u(t)$ are checked for compliance with the limiting functions at every instant of time before feeding as inputs to the plant. The plant inputs $\hat{u}(t)$ and its past outputs $y(t)$ are used by the online model to provide further predictions of the plant behaviours. The controller utilizes these predictions to train and calculate the next set of plant inputs. The neural network approximation of this autoregressive structure and exogenous inputs model is given in Eq. (3)

$$
\begin{aligned}
& y_{m}\left(t \mid \theta_{m}\right)=F_{1 s}\left\{\sum_{j=1}^{l_{11}}\left\{W_{11 j} G_{1 j}\left\{\sum_{s=1}^{l_{12}}\left\{W_{12 i j} x_{1 i}(k)+W_{120}\right)\right\}\right\}+W_{110}\right\} \\
& \theta_{m}(t)=\left[W_{11 j s}, W_{110}, W_{12 i j}, W_{120}\right] \\
& \phi_{m}(t)=x_{1 i}(t)
\end{aligned}
$$

Where $y_{m}$ is the network predicted output, $w_{11 \text { js }}$ and $w_{12 i j}$ are the output layer and hidden layer weights matrices, $F_{1 s}$ and $G_{1 j}$ are the activation functions of the neurons ion the output and the hidden layers respectively. $l_{11}$ and $l_{12}$ are the number of neurons in the two layers, $W_{110}$ and $W_{120}$ are the two layers and $x_{1 i}$ is the network input vector, $i, j$ and $s$ are the indices to the input vector, hidden nodes and output nodes in the network respectively. The internal model network in the double neural network controller be represented by Eq. (3), the model repressor function includes the past input and output terms and the present input terms. Where, $y_{2 i}(t)$ is the present input to the model network and $y_{m}(t-1) \ldots$ are the past outputs from it.

$$
\phi_{m}(t)=x_{1 i}(t)=\left[y_{2 i}(t), y_{2 i}(t-1), \ldots, y_{m}(t-1) \ldots\right]
$$


The controller network is an online trained auto regressive structure and exogenous inputs model based recurrent neural network. Let the weights for controller network be represented as a vector $\theta_{c}$ and the repressor matrix by $\phi_{c}$. The predicted output form the controller network is given by Eq. (6).

$$
\begin{aligned}
& y_{2 i}\left(t \mid \theta_{c}\right)=F_{2 i}\left\{\sum_{j=1}^{l_{21}}\left\{W_{21 j i} G_{2 j}\left\{\sum_{s=1}^{l_{22}}\left\{W_{22, j j} y_{i n}(t)+W_{220}\right\}\right\}\right\}+W_{210}\right\} \\
& \theta_{c}(t)=\left[W_{21 j i}, W_{210}, W_{22 . j j}, W_{220}\right] \\
& \phi_{c}(t)=y_{i n}(t)
\end{aligned}
$$

$W_{22 j s}$ and $W_{21 i j}$ are the output layer and hidden layer weights matrices, $F_{2 i}$ and $G_{2 j}$ are the activation functions of the neurons ion the output and the hidden layers respectively. $l_{21}$ and $l_{22}$ are the number of neurons in the two layers, $W_{210}$ and $w_{220}$ are the two layers and $y_{i n}$ is the controller network input vector which is obtained from a combination of the commanded reference input and the predicted plant outputs form the online network model.

$$
y_{\text {in }}(t)=F\left[\hat{y}(t), \hat{y}(t-1), \ldots, y_{r e f}(t), y_{r e f}(t-1) \ldots\right]
$$

Then, from Eq. $(3,4)$ the model network output can be expressed as the function of the effective input vector given by Eq. (9)

$$
y_{m}\left(t \mid \theta_{m}\right)=f\left(\theta_{m} \cdot g\left(\theta_{c}(t) \cdot y_{\text {in }}(t)\right)\right)
$$

The equilibrium values for each of the control element are recorded and corrections to these values are provided to achieve the desired response. For example, the trimmed flight condition can be considered as the equilibrium position for an aircraft in flight. The aircraft can be trimmed at different flight conditions and at each time the trimmed values are utilized in the control process. The equilibrium values are used with the reference inputs to obtain the effective input vector into the double neural network controller. The effective input vector is given by Eq. (10)

$$
y_{\text {in }}(t)=y_{\text {ref }}(t)-y_{\text {eq }}(t)+\hat{y}(t)
$$

Where $y_{e q}(t)$ the equilibrium is value and $\hat{y}(t)$ is the predicted output from the online network model. The controller network outputs $y_{2 i}\left(t \mid \theta_{c}\right)$ from Eq. (6) are validated through the model network at every iteration of training. The augmentation to the plant input corresponding to that of the controller input is given by Eq. (11)

$$
u(t)=u_{N}(t)-u(t-1)+u_{e q}(t)
$$

Where $\mathrm{u}(\mathrm{t})$ is the current plant input, $u_{N}(t)=y_{2 i}\left(t \mid \theta_{c}\right)$ is the online controller network output and $u_{e q}(t)$ is the equilibrium value of the plant input corresponding to $y_{e q}(t)$ value.

\subsection{Online Training}

The goal of training is to obtain the most suitable values of weights to attain the desired performance through repetitive iterations. Here not only the mean square error(MSE) between 
the effective input $y_{i n}$ and the validation output from the internal model network $y_{m}$ as a function of the controller network weights, but also the instantaneous error between the reference input to the controller and the predicted output from the external identifier model are minimized.

The $E_{\text {err } 1}$ as a function of the controller network weights $\theta_{c}$, to be minimized is given by Eq. (12).

$$
E_{e r r 1}\left(\theta_{c}, t\right)=\frac{1}{M} \sum_{i=1}^{M}\left(y_{i n}(t)-y_{m}\right)^{2}
$$

Instantaneous error of the overall adaptive system is calculated from the controller reference input and the online network identifier model output shown by Eq. (13)

$$
E_{\text {err } 2}\left(\theta_{c}, t\right)=\left(y_{\text {ref }}(t)-\hat{y}(t)\right)
$$

The instantaneous error function initiates training in the controller network when the plant deviates from the reference input. It is to be noted that the output of the internal model $y_{m}$ changes every iteration during training of the controller, where $\hat{y}(t)$ remains constant during training.

The two error functions considered in Eq. $(12,13)$ have different characteristics. The $E_{\text {err } 1}$ function indicates error in the inner loop of the controller with respect to the internal model. The instantaneous error indicates the overall discrepancy in the adaptive system through the outer loop. It provides the difference between the online identifier model prediction and the commanded reference input. The instantaneous error is checked at every sample period and is retained for the duration of the training cycle. With the two error functions in the performance index of training, overall performance of the dual network controller is enhanced.

\section{Experiments for Eight-Rotor MAV Autonomous Flight}

The application of the double neural network controller is considered in this section. To compare with the performance of the proposed double neural network controller with the conventional techniques, Proportional-Integral-Differential (PID) controllers with gains tuned for selective flight conditions are designed. The results based on the proposed technique are shown to be superior to the PID technique under the influence of Eight-Rotor variations and external disturbances. The overall control architecture for the Eight-Rotor MAV is presented here too.

\subsection{Eight-Rotor MAV Flight Control Structure}

This section presents the devices and their connections for the overall architecture of Eight-Rotor helicopter control system, which is equipped with a RF receiver, Micro Controller Unit (ARM S3C2410), eight motor's power boards and several sensors to provide a stable autonomous system. The block diagram of this architecture is provided by Figure 4. 


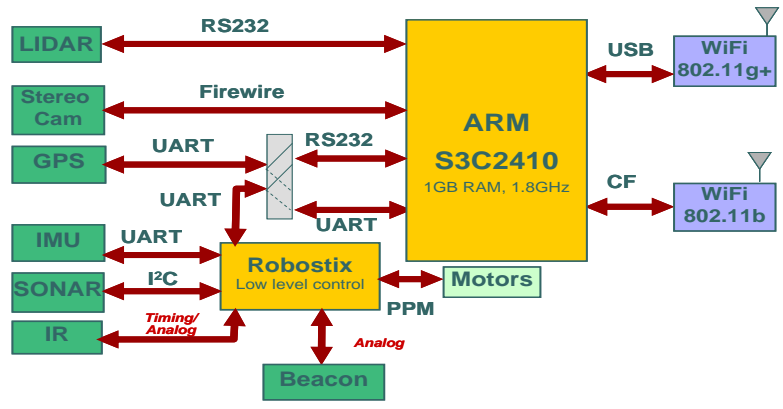

Figure 4. The Block Diagram of the Architecture for Control System

The GPS module represents the device which provides information about the Eight-Rotor's global position. It is physically built in the IMU, but its information is not provided by inertial sensor, it can be connected as a feedback for the desired outdoor global trajectory.

The IMU module represents the physical device which provides information about EightRotor's attitude and heading. It is composed of three accelerometers, three magnetometers, three gyroscopes, a barometer and a temperature sensor. Thanks to all these components, the IMU calculates the roll-pitch-yaw angles $(\varphi, \theta, \psi)$ and it sends them to a MCU's UART. The communication is provided through a digital RS-232 interface. Furthermore, thanks to the three gyroscopes, it is possible to have a feedback about the Eight-Rotor's angular speeds in the fixed-body frame (p,q,r).The IMU is therefore an essential device for Eight-Rotor helicopter.

The SONAR\&IR module represents the devices involved to estimate the distance from the Eight-Rotor to an obstacle in a certain direction. Two different systems have been mounted: the SONAR and the IR module. The SONAR detects the distance of an obstacle thanks to ultrasound waves. The IR module has a narrower beam and is connected to the MCU thanks to a MCU's ADC since the information is analogy. These devices are used to estimate the height of the Eight-Rotor from the ground and to provide information on the availability of the space around itself.

\subsection{PID Controller for Eight-Rotor MAV}

The Eight-Rotor attitude control includes the Roll angle, Pitch angle and Yaw angle control, here the PID controller adopted to control the Euler angle. An optimization method for tuning provides the most suitable combination of gains based on the actuator constraints and the output constraints. These constraints are in the form of upper bounds on the peak overshoot, settling time and steady state error form the response to a step input, a block diagram of the attitude autopilot is shown in Figure 5. The response of the plant model for a combination of ramp and step inputs with this PID autopilot is shown in Figure 6. The result shows a close match between the plant response and the reference input.

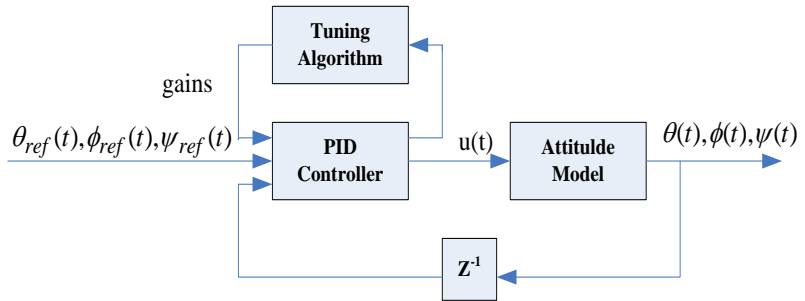

Figure 5. PID Controller based of Attitude Autopilot for Eight-Rotor MAV 


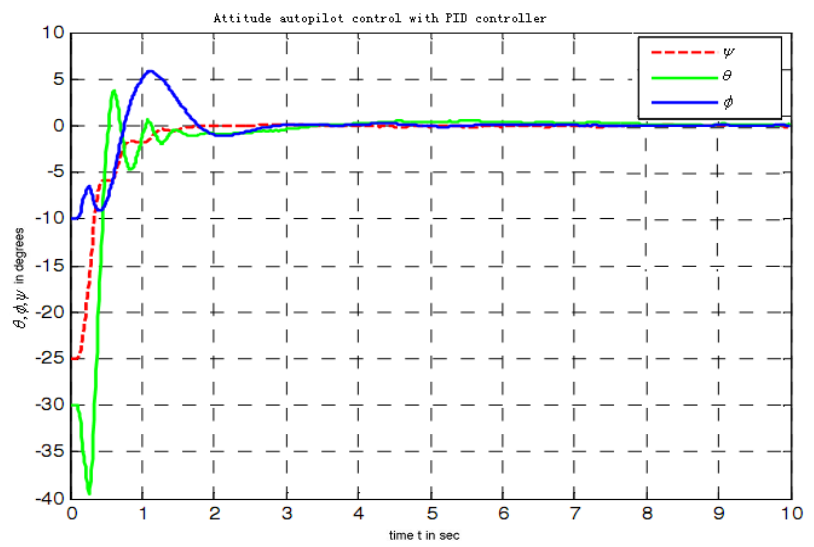

Figure 6. Attitude Autopilot Control for Eight-Rotor MAV with PID Controller

\subsection{Double Neural Network Controller for Eight-Rotor MAV}

PID controllers are efficient in single-loop feedback control; however, classical control techniques do not address the issue of coupling exhibited by multi-variable systems such as the Eight-Rotor MAV. The results form comparison of PID controller with adaptive double neural network controller is presented here. It is observed that the PID controller is incapable to maintain the commanded reference when the plant is subjected to windy conditions; the PID controllers also fail to cope with the variation in the dynamics of the Eight-Rotor MAV.

To solve the issues shown by using PID controllers, the adaptive double neural network controller is presented here. Simulation results using adaptive double neural network controller are compared with those from the PID controller for varied flight conditions and under the influence of external disturbance. To test the controller in simulation for robustness, the sensor noise and effects of gusts are included. An adaptive double neural network based attitude autopilot is designed for Eight-Rotor MAV with aid of the block diagram shown in Figure 7. The $\theta$ angle control by using double neural network controller and PID controller, from the Figure 8 we can see that the double neural network controller performs faster than PID controller and has less overshoot. The sensor noise is a band limited white noise, whereas the vertical gusts are included in the form of small bursts at discrete times. A typical result for tracking $[13,14]$ a given $\theta$ in the presence of these two disturbances is shown in Figure 9.

Adaptive double neural network controller

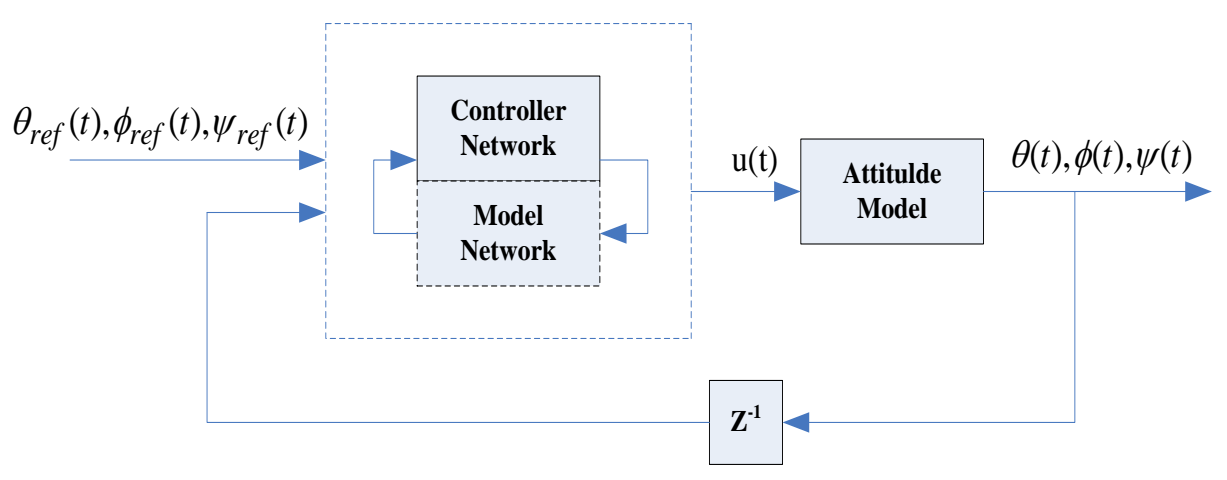

Figure 7. Adaptive Double Neural Network Controller based Attitude Control for Eight-Rotor MAV 


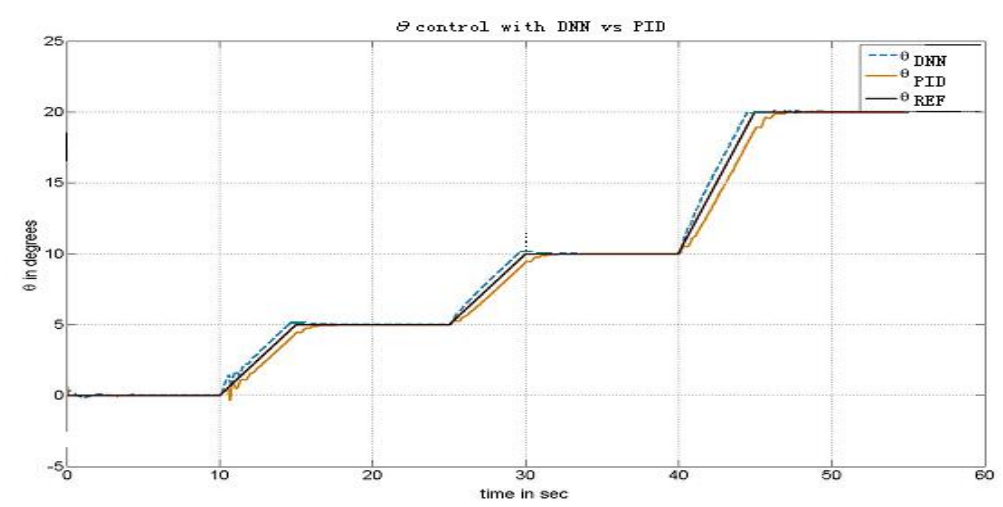

Figure 8. ${ }^{\theta}$ Control Compared by Using Double Neural Network Controller vs PID Controller
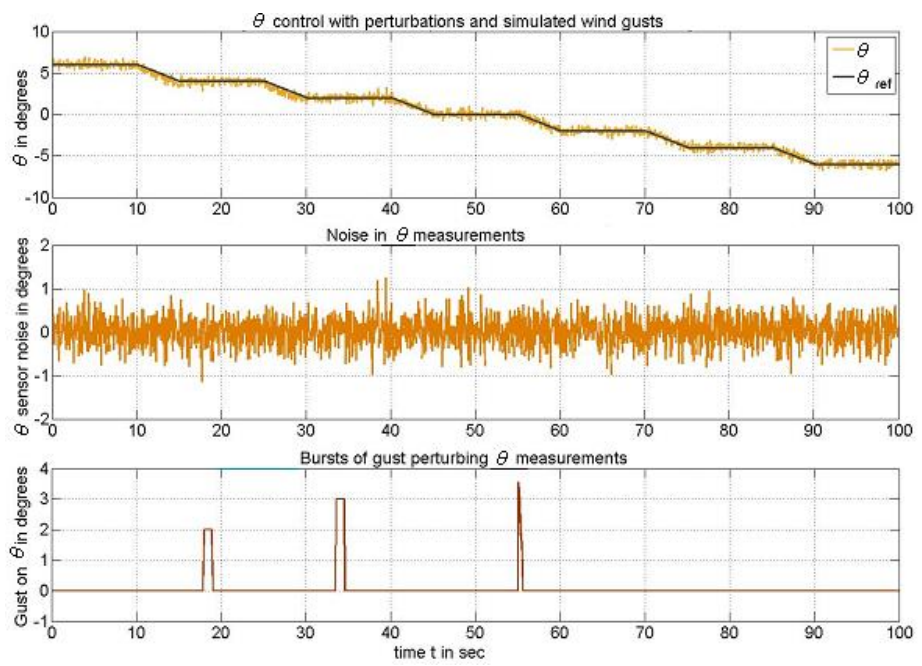

Figure 9. Tracking a Given $\theta$ in the Presence of Perturb and Wind Gusts

\section{Conclusion}

In this paper, an new Eight-Rotor MAV which provides excellent manoeuvrability and maintains a simple mechanical construction without the need for control surfaces as on an airplane or variable-pitch propellers as on helicopter. This mechanical simplicity comes at a cost of increased controller complexity. The Eight-Rotor MAV is inherently unstable as they are highly sensitive to even the smallest differences in rotor speeds. Hence, here provides the dynamic modelling of the Eight-Rotor MAV and also design the adaptive dual neural network controller for this MAV autonomous flight.

The initial autonomous control aims to stabilize the craft and outputs rotor speeds based on the requested attitude. The controller is developed in three parts around each of the variables, initially training them to achieve the results be superior to the PID controller. The experimental results show that stable control is achieved through this control architecture. In addition, the results show that the adaptive dual neural network controller outperforms the conventional PID controller due to its fast adaptive qualities in the presence of sensor measurement noise and plant parameters variation. 


\section{References}

[1] P. McKerrow, "Modelling the Draganflyer four-rotor helicopter", Proceedings of the IEEE International Conference on Robotics and Automation, (2004), pp. 3596-3601.

[2] P. Pounds, R. Mahony, P. Hynes, et al., "Design of a four-rotor aerial robot", Proceedings of Australian Conference on Robotics and Automation, pp. 145-150, (2002).

[3] P. McKerrow, "Modelling the Draganflyer four-rotor helicopter", Proceedings of the IEEE International Conference on Robotics and Automation, (2004), pp. 3596-3601.

[4] A. Tayebi and S. McGilvray, "Attitude stabilization of a four-rotor aerial robot", 43rd IEEE Conference on Decision and Control, (2004), pp. 357-364.

[5] P. McKerrow, "Modelling the Draganflyer four-rotor helicopter", In proceedings of the 2004 IEEE International Conference Robotics and Automation, (2004), pp. 132-141.

[6] J. T. Spooner and M. Passino, "Stable adaptive control using fuzzy systems and neural networks", IEEE Transactions on Fuzzy Systems, vol. 4, no. 3, (1999), pp. 339-359.

[7] C. H. Hong, K. C. Choi and B. S. Kim, "Applications of Adaptive Neural Network Control to an Unmanned Airship", International Journal of Control, Automation, and Systems, vol. 7, no. 6, (2009), pp. 911-917.

[8] L. Di, C. Xiangjian and B. Yue, "Reseach on the Modeling and Simulation for Eight-Rotor MAV", In 2010 IIS 2nd International Conference on Signal Processing, Robotics and Automation, vol. 2, (2010), pp. 412-1412-3.

[9] H. Abid, M. Chtourou and A. Toumi, "An Indirect Model Reference Robust Fuzzy Adaptive Control for a Class of SISO Nonlinear Systems",International Journal of Control, Automation, and Systems, vol. 7, no. 6, (2009), pp. 982-991.

[10] R. Ordonez and K. Passino, "Stable multi-input multi-output adaptive fuzzy/neural control", IEEE Transactions on Fuzzy Systems, vol. 7, no. 3, (1999), pp. 345-353.

[11] M. M. Polycarpou, "Stable adaptive neural control scheme for nonlinear systems", IEEE Transactions on Automatic Control, vol. 41, no. 3, (1999), pp. 447-451.

[12] S. Bouabdallah and A. Noth, "Siegwart R.PID vs. LQ Control Techniques Applied to an Indoor MicroQuadrotor", IEEE International Conference on Intelligent Robots and Systems, (2004), pp. 546-551.

[13] Y. Sundararajan, N. Sundararajan, L. P. Sratchandran, et al., "Neuro-controller design for nonlinear fighter aircraft maneuver using fully tuned RBF networks", Automatica, vol. 37, (2001),pp. 1293-1301.

[14] D. Dathbun, S. Kragelund, A. Pongpunwattana, B. Capozzi, et al., "An evolution based path planning algorithm for autonomous motion of a UAV through uncertain environments", In 21 st digital avionics systems conference, vol. 2, (2002), pp. 8D2-1-8D2-12.

\section{Authors}

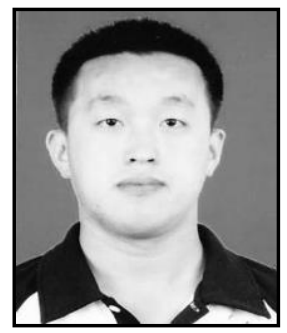

Di Li, studies embedded operation system and control, and he is a Ph.D. graduated from the Department of Mechanical and Electronic Engineering, Chang Chun Institute of optics fine mechanics and physics Chinese academy of sciences, China, in 2012.His interests include embedded operation system, image processing and Micro aircraft vehicle.

Email: lidi19821111@163.com 